ARTICLE

https://doi.org/10.1038/s41467-020-15299-5

\title{
Exfoliated near infrared fluorescent silicate nanosheets for (bio)photonics
}

Gabriele Selvaggio', Alexey Chizhik², Robert Nißler', Ilyas Kuhlemann (D) ${ }^{1}$, Daniel Meyer ${ }^{1}$, Loan Vuong ${ }^{3}$,

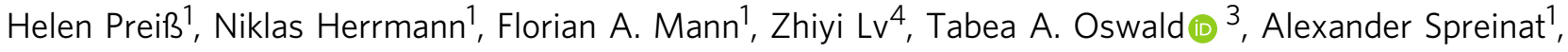
Luise Erpenbeck ${ }^{5}$, Jörg Großhans ${ }^{4}$, Volker Karius ${ }^{6}$, Andreas Janshoff ${ }^{1}$, Juan Pablo Giraldo (i) ${ }^{7}$ \&

Sebastian Kruss (iD ${ }^{1 凶}$

Imaging of complex (biological) samples in the near-infrared (NIR) is beneficial due to reduced light scattering, absorption, phototoxicity, and autofluorescence. However, there are few NIR fluorescent materials known and suitable for biomedical applications. Here we exfoliate the layered pigment $\mathrm{CaCuSi}_{4} \mathrm{O}_{10}$ (Egyptian Blue, EB) via ball milling and facile tip sonication into NIR fluorescent nanosheets (EB-NS). The size of EB-NS can be tailored to diameters $<20 \mathrm{~nm}$ and heights down to $1 \mathrm{~nm}$. EB-NS fluoresce at $910 \mathrm{~nm}$ and the fluorescence intensity correlates with the number of $\mathrm{Cu}^{2+}$ ions. Furthermore, EB-NS display no bleaching and high brightness compared with other NIR fluorophores. The versatility of EB-NS is demonstrated by in-vivo single-particle tracking and microrheology measurements in Drosophila melanogaster embryos. EB-NS can be uptaken by plants and remotely detected in a low-cost stand-off detection setup. In summary, EB-NS have the potential for a wide range of bioimaging applications.

\footnotetext{
${ }^{1}$ Institute of Physical Chemistry, University of Göttingen, Göttingen 37077, Germany. ${ }^{2}$ Third Institute of Physics, University of Göttingen, Göttingen 37077 , Germany. ${ }^{3}$ Institute of Organic and Biomolecular Chemistry, University of Göttingen, Göttingen 37077, Germany. ${ }^{4}$ Institute of Developmental Biochemistry, Medical School, University of Göttingen, Göttingen 37077, Germany. ${ }^{5}$ Department of Dermatology, Venereology and Allergology, University Medical Center Göttingen, Göttingen 37075, Germany. ${ }^{6}$ Department of Sedimentology and Environmental Geology, Geoscience Center, University of Göttingen, Göttingen

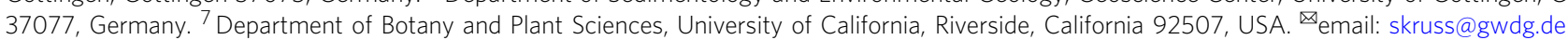


Fimatis luorescence imaging provides important insights into the structure, function, and dynamics of biological samples ${ }^{1,2}$. Imaging in the near-infrared (NIR) spectral range (800-1700 nm) promises higher tissue penetration, higher contrast, and lower phototoxicity due to reduced NIR light scattering and absorption ${ }^{3-5}$. However, these approaches are limited by the scarcity of NIR fluorescent materials. NIR fluorescent organic dyes such as indocyanine green (ICG) bleach and are therefore not suitable for long-term imaging ${ }^{6,7}$. In contrast, nanomaterials provide beneficial photophysical properties such as ultra-high photostability that would enable tracking in living systems without time constrains. NIR fluorescent nanomaterials include InAs quantum dots, lanthanide-doped nanoparticles, or semiconducting single-walled carbon nanotubes (SWCNTs) ${ }^{8-12}$. For example, SWCNTs have been used as building blocks for NIR imaging and as fluorescent sensors that detect small signaling molecules, proteins, or lipids ${ }^{13-19}$. They can be chemically tailored and have been used to reveal spatiotemporal release patterns of neurotransmitters from single cells $2,15,20-22$. However, most NIR fluorescent nanomaterials often have low quantum yields, lack biocompatibility, or are restricted to certain emission/ excitation wavelengths. Therefore, there is a major need for novel NIR fluorescent and biocompatible nanomaterials for sophisticated applications such as long-time single-particle tracking in organisms or multiscale bioimaging such as stand-off detection in plants $^{23,24}$.

One of the first colored pigments created by mankind is the calcium copper silicate called Egyptian Blue $\left(\mathrm{CaCuSi}_{4} \mathrm{O}_{10}, \mathrm{~EB}\right)$, which has been synthesized and used as early as 2500 BC in Ancient Egypt ${ }^{25}$. Current ancient works of art decorated with EB have lost none of their vibrant color, a testimony to the remarkable chemical stability of this compound. Interestingly, bulk EB displays NIR fluorescence, which was only recently identified ${ }^{26,27}$ and attributed to a ${ }^{2} \mathrm{~B}_{2 g^{-}}{ }^{2} \mathrm{~B}_{1 \mathrm{~g}}$ electronic transition of the copper ion that ranges from 910 to $930 \mathrm{~nm}^{26,28}$. Bulk EB shows a remarkable high quantum yield of $10.5 \%$ for a NIR emitter compared with SWCNTs, quantum dots, metal nanoclusters, and FDA-approved fluorophores such as ICG $^{5,28}$. Recently, micrometer-sized monolayer sheets of EB were isolated by stirring in hot water for several days ${ }^{29,30}$. However, the remarkable properties of EB have not been explored for developing NIR luminescent nanomaterials for bioimaging applications. The layered structure of EB suggests that exfoliation procedures known from other two-dimensional (2D) materials including graphene and transition metal chalcogenides could efficiently exfoliate $i^{31-34}$. Such $2 \mathrm{D}$ materials have been shown to possess promising optoelectronic properties and are a rich playground for physics and chemistry ${ }^{35}$.

Herein, we use a facile tip sonication technique to exfoliate $\mathrm{CaCuSi}_{4} \mathrm{O}_{10}$ (EB) nanosheets (EB-NS). This procedure allows one to control the nanomaterial size/thickness and retain the unique NIR fluorescent properties of macroscopic $\mathrm{CaCuSi}_{4} \mathrm{O}_{10}$. We report the photophysical properties of EB-NS and investigate how NIR fluorescence scales with nanosheet size. Furthermore, we demonstrate the use of this material for in-vivo NIR microscopy and stand-off detection.

\section{Results}

Exfoliation of Egyptian Blue into nanosheets. The size of a nanomaterial determines its properties and interactions with the environment. For fluorescence imaging in cells or whole organisms, fluorophores should be as small as possible to not perturb the system. Exfoliation into 2D sheets is one step but it is also important to decrease the lateral size into the nanoscale. Therefore, we first reduced the size of EB by planetary ball (PB) milling and then exfoliated EB-NS via tip sonication, which allowed for the controlled decrease in height and diameter with sonication time (Fig. 1a and Supplementary Fig. 1).

Milling techniques such as $\mathrm{PB}$ are routinely employed for silicates $^{36}$, whereas tip sonication is a widely used method to disperse nanomaterials such as carbon nanotubes ${ }^{37}$. Tip sonication for $6 \mathrm{~h}$ in isopropanol yielded nanosheets of lateral sizes between 20 and $300 \mathrm{~nm}$, and heights of around $1-13 \mathrm{~nm}$ (Fig. 1b-d). Atomic force microscopy (AFM) images (Fig. 1b, c) indicate that EB height correlated linearly with diameter (Fig. 1d). The height profiles of EB-NS (Fig. 1e, f) typically contain a peak, which can be explained by the convolution through the AFM tip. The smallest observed height $(\approx 1 \mathrm{~nm})$ corresponds to the monolayer of EB bulk material reported in literature ${ }^{29,38,39}$ (Supplementary Fig. 2), but the exact height measured with AFM might vary depending on hydration and underlying substrate morphology ${ }^{29}$. To further purify EB-NS samples, we typically used syringe cut-off filters $(0.20 \mu \mathrm{m} / 0.45 \mu \mathrm{m})$ to remove the remaining larger particles from the samples.

Photophysical properties of EB-NS. In a next step, we explored how reducing the size and dimensionality of EB into the nanoscale regime affects its luminescence properties relative to macroscopic EB powder ${ }^{27}$. Fluorescence quantum yields of onedimensional materials such as SWCNTs have been shown to decrease with size, probably due to exciton diffusion and their collision with SWCNT ends ${ }^{40}$. Interestingly, EB fluorescence spectra did not change/shift with longer sonication times (Fig. 2a) corresponding to smaller dimensions (Fig. 1). EB-NS are extremely photostable as evidenced by the constant fluorescence emission intensity over several hours compared with the rapid bleaching of a typical organic dye (Fig. 2b). These measurements were performed on an organic dye (Rhodamin B) and on EB-NS, which were adsorbed on a glass surface under continuous excitation with a $561 \mathrm{~nm}$ laser (100 $\mathrm{mW}$ total power output) for $>2 \mathrm{~h}$. In addition, EB-NS show no change or shift in fluorescence emission in the presence of small redox active molecules that are known to affect the fluorescence of many dyes and fluorescent nanomaterials (Supplementary Fig. 3). EB-NS fluorescence is characterized by a large Stokes shift with a single emission maximum at $\approx 910 \mathrm{~nm}$ and an absorption maximum at $\approx 630 \mathrm{~nm}$, as shown in the $2 \mathrm{D}$ excitation-emission spectrum and in the reflectance spectrum (Fig. $2 \mathrm{c}$ and Supplementary Fig. 4). EB-NS have a similar zeta potential $(-22 \mathrm{mV})$ to spherical silica nanoparticles, which highlights that they can be dispersed and applied in aqueous solutions (Supplementary Fig. 5) ${ }^{41}$.

To estimate the number of luminescent centers in a single EBNS, we performed single-particle fluorescence saturation measurements of EB-NS using scanning confocal microscopy and pulsed laser excitation $(20 \mathrm{MHz}$ pulse rate). Only EB-NS below the resolution limit were studied (Fig. 2d, Supplementary Fig. 6, and Supplementary Table 1). Larger single sheets showed higher fluorescence intensities and could be distinguished from resolution-limited ones (see also Figs. 3 and 4). This finding complies with the hypothesis that the number of luminescent centers changes with the size of EB-NS. To better understand how fluorescence scales with excitation intensity, the laser power was stepwise increased and decreased, while fluorescence intensity was recorded. It was not possible to reach the emission saturation plateau with the maximum laser intensity. However, the nonlinear dependence between fluorescence intensity and laser power allowed us to fit the data with a fluorescence brightness saturation function $(I)$ :

$$
I=I_{\mathrm{sat}} \frac{P_{\mathrm{exc}}}{P_{\mathrm{exc}}+P_{\mathrm{sat}}},
$$


a

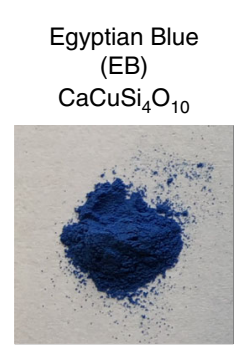

b

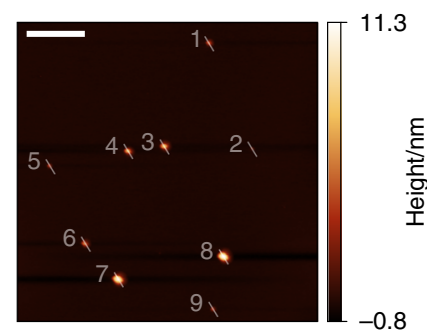

2D EB nanosheets (EB-NS)

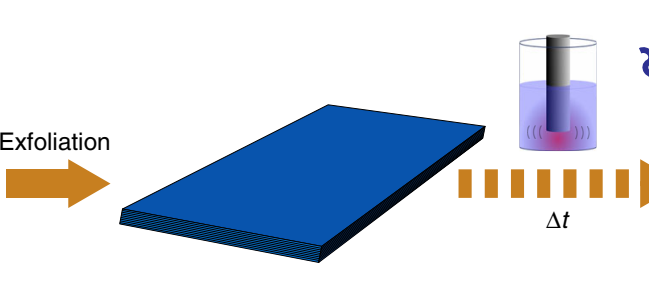

C

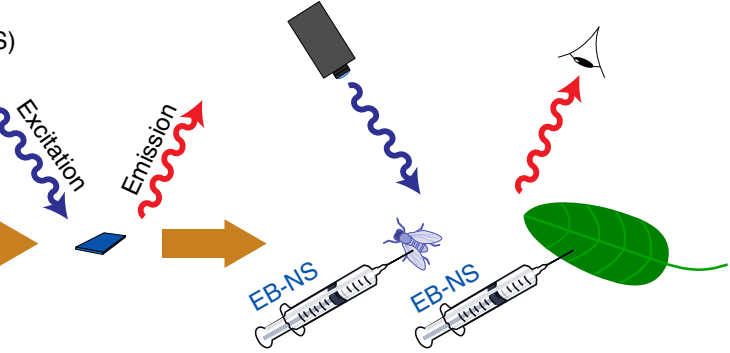

d
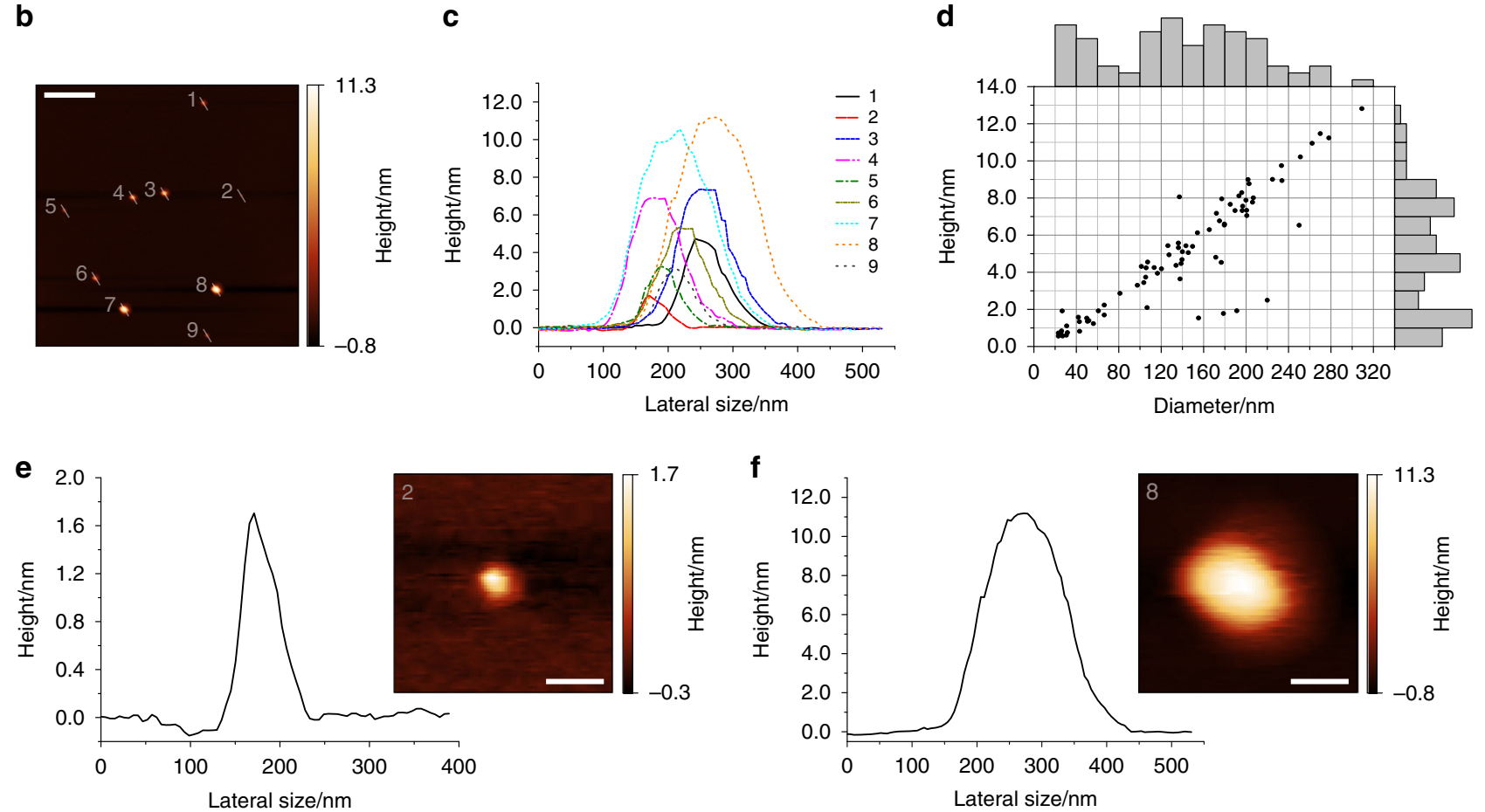

Fig. 1 Exfoliation of Egyptian Blue (EB) into Egyptian Blue nanosheets (EB-NS). a EB bulk powder ( $\mathrm{CaCuSi}_{4} \mathrm{O}_{10}$, photograph taken in our lab) is exfoliated into EB-NS via tip sonication to study their near-infrared (NIR) photophysical properties. These nanostructures are furthermore used for in-vivo NIR imaging in plants and Drosophila embryos. b Representative AFM image of EB-NS. Scale bar $=1 \mu \mathrm{m}$. c Corresponding height profiles (highlighted by a white line in b). $\mathbf{d}$ Height and diameter distribution after $6 \mathrm{~h}$ of tip sonication. The bars at the axis are histograms. $n=78$ EB-NS. e, $\mathbf{f}$ Height profiles and magnified images of the smallest and largest nanosheets shown in $\mathbf{b}$. Scale bar $=100 \mathrm{~nm}$.

where $P_{\text {exc }}$ is the excitation power, $P_{\text {sat }}$ is the saturation power, and $I_{\text {sat }}$ is the nanoparticle luminescence intensity that can be detected at the saturation excitation power ${ }^{42}$.

The obtained saturation values correspond to a fluorescence emission of $122 \times 10^{3}$ to $1250 \times 10^{3}$ photons s $^{-1}$. By taking into account factors such as the average lifetime of the excited state $(\approx 100 \mu$ s; Supplementary Fig. 7$)$, the quantum yield $\left(\approx 0.1^{28}\right)$ of bulk EB, the efficiency of the optical setup (see Supplementary Methods for an accurate calculation and Supplementary Table 1), it was thus possible to estimate the number of luminescent centers $N(122-1250)$.

From the crystal structure of EB, it follows that there are 3.8 $\mathrm{Cu}^{2+}$ ions $\mathrm{nm}^{-2}$ of a single-layer EB-NS ${ }^{43}$ (Supplementary Fig. 2). If every $\mathrm{Cu}^{2+}$ ion serves as luminescent center, these numbers would be in agreement with around $6-18 \mathrm{~nm}$ large (squared) single-layer EB-NS. From a resolution-limited EB-NS image, we cannot derive the lateral size and height directly. Therefore, these numbers are rather a minimum size that is in agreement with the experiment. Nevertheless, these data suggest that a large proportion, if not all, of $\mathrm{Cu}^{2+}$ ions act as luminescent centers. Furthermore, EB-NS showed no change of fluorescence intensity at different polarization directions (Fig. 2e) compared with a typical organic dye (Atto 488). Similar to NIR emitting lanthanide complexes, bulk EB has a long fluorescence lifetime ranging from $107 \mu$ s to $142 \mu \mathrm{s}$ at room temperature 28,44 and this behavior is retained in EB-NS (Supplementary Fig. 7). The long fluorescence lifetime is most likely a consequence of the parityforbidden nature of the transition, as the local $\mathrm{D}_{4 \mathrm{~h}}$ symmetry of the $\mathrm{Cu}^{2+}$ ion in EB features an inversion center ${ }^{27}$.

NIR fluorescence imaging of single EB-NS. Larger EB-NS typically exhibit a layered structure as observed in scanning electron microscopy (SEM) images (Fig. 3a, b and Supplementary Fig. 8). PB milling followed by exfoliation via tip sonication enabled us to continuously control the decrease in size of EB from large macroscopic particles to micrometer large structures down to EB-NS. These large and layered EB-NS can be imaged by a NIR fluorescence microscope, but do not show a uniform fluorescence intensity profile, indicating that differences in thickness or geometry might affect fluorescence emission intensities. EB-NS smaller than the resolution limit of light microscopy can also be imaged in the NIR (Fig. 3c, d, e). To prove this point, both a Si-based and an InGaAs camera were employed in a wide-field setup. The laser intensities in 

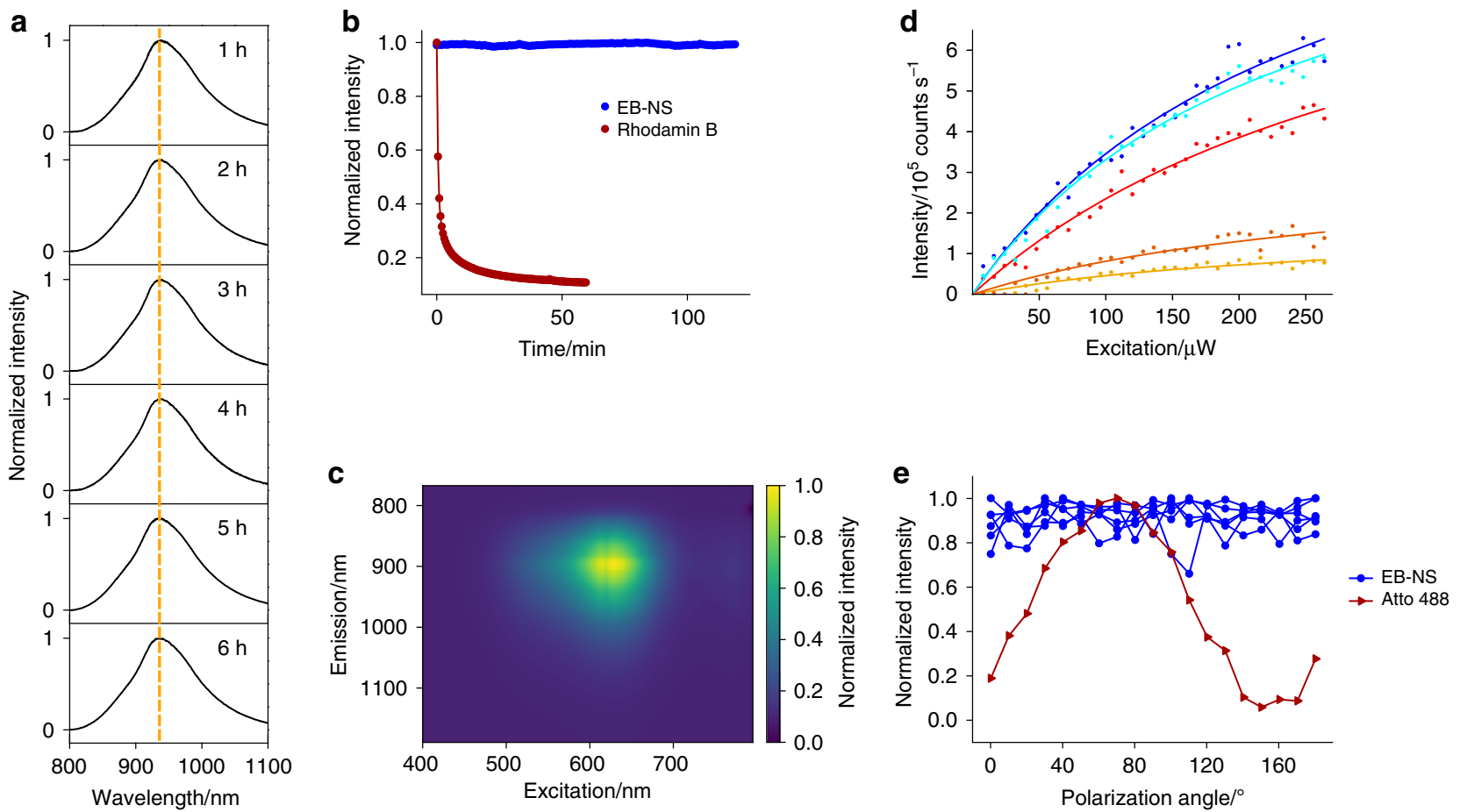

Fig. 2 NIR fluorescence properties of EB-NS. a Normalized NIR fluorescence spectra of EB-NS after different tip sonication times show no wavelength shifts. b EB-NS fluorescence (NIR) under continuous laser excitation ( $561 \mathrm{~nm}, 100 \mathrm{~mW}$, corresponding to about $1 \mathrm{~W} \mathrm{~cm}^{-2}$ ) does not bleach. In contrast, the fluorescent dye Rhodamin B (in the visible) quickly bleaches. c 2D excitation-emission spectrum of EB-NS. d Fluorescence saturation curves of different EBNS (size below the resolution limit). e EB-NS display no fluorescence polarization. The dye Atto 488 is used as reference due to its distinct fluorescence polarization.
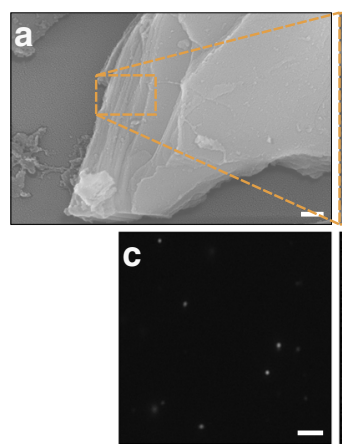
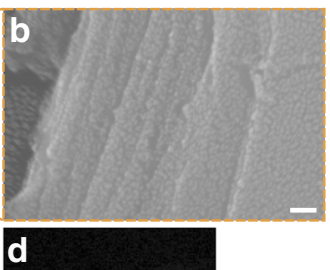
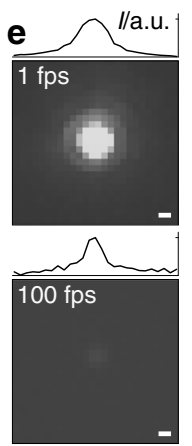
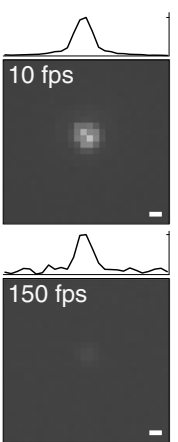

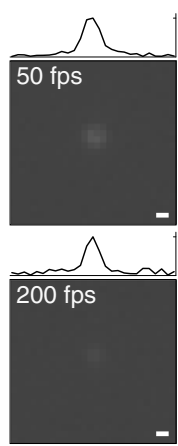

Fig. 3 SEM and NIR imaging of EB-NS. a, b Scanning electron microscopy (SEM) images of a larger EB particle. The blow-up clearly shows the typical lamellar structure of this material. Scale bar $=200 \mathrm{~nm}$ and $50 \mathrm{~nm}$ (respectively). c, d NIR images of EB-NS taken with a Si-based camera at increasing magnification show that single EB-NS can be resolved via NIR fluorescence microscopy down to the resolution limit of light microscopy. Excitation wavelength $=561 \mathrm{~nm}$. Scale bar $=5 \mu \mathrm{m}$ and $1 \mu \mathrm{m}$ (respectively). e NIR images of a single EB-NS with a diameter below the resolution limit $(\approx 500 \mathrm{~nm})$ are captured with an InGaAs camera, optimized for NIR imaging at different frame rates up to $200 \mathrm{fps}$. The upper traces show the intensity profile of the image normalized to the maximum value. Excitation wavelength $=561 \mathrm{~nm}$. Scale bar $=500 \mathrm{~nm}$.

this setup corresponded to the left region $(<10 \mu \mathrm{W})$ in the confocal setup used for saturation experiments (Fig. $2 \mathrm{~d}$ ). Si-based cameras have the highest quantum yield in the visible range and not the NIR, which limits the possible speed of imaging to around $50 \mathrm{fps}$ (Fig. 3c, d and Supplementary Fig. 9). In contrast, with an InGaAs camera, resolution-limited EB-NS could be imaged at video rates up to $200 \mathrm{~Hz}$ (Fig. 3e). These images clearly demonstrate that the NIR fluorescence properties of macroscopic EB particles are retained in nanosheets.

Correlation between size and fluorescence intensity of EB-NS. The previous observations raise the question of how fluorescence intensity scales with size and if there is a lower limit, which is also relevant for potential bioimaging applications. Unfortunately, NIR microscopy is not able to directly assess the EB-NS size below the resolution limit. The resolution limit of light microscopy is described by the Abbe law $(\lambda / 2 \approx 910 \mathrm{~nm} / 2 \approx 450 \mathrm{~nm})$ and an exemplary resolution-limited single EB-NS is shown in Fig. 3e. The photon counting experiments from Fig. $2 \mathrm{~d}$ show that there are many luminescent $\mathrm{Cu}^{2+}$ centers in one EB-NS, but because of the resolution limit it is not possible to assess the actual size. To provide an unambiguous answer, a correlative method that measures both size and fluorescence intensity of the same single nanosheet is required.

To understand whether and how nanomaterial fluorescence intensity is affected by size, we performed two independent 
a

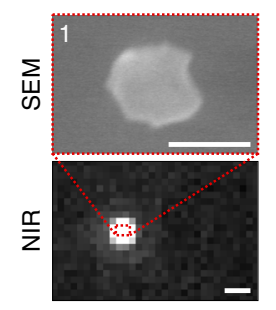

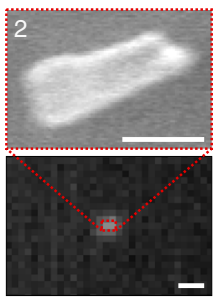

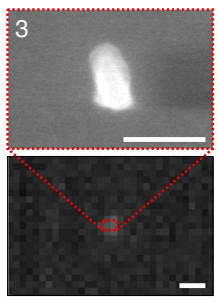

b

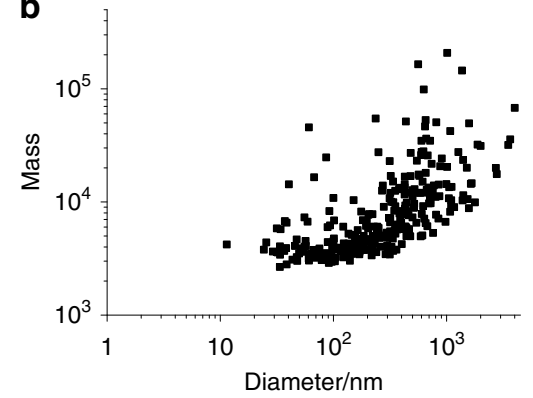

Fig. 4 Correlative measurements of size and fluorescence intensity of EB-NS. a SEM and corresponding NIR images of resolution-limited EB-NS. Scale bar $=200 \mathrm{~nm}$ and $1 \mu \mathrm{m}$ (respectively). b Fluorescence intensity as a function of hydrodynamic diameter of EB-NS obtained by the corrected Stokes-Einstein equation. Mass stands for the total integrated brightness of the particle, and the maximum value along each trajectory was taken. From this analysis, one notices that even the smallest EB-NS $(\approx 10 \mathrm{~nm})$ still fluoresce. The general trend indicates that EB-NS of larger size are brighter. $n=292$ EB-NS.

correlative analyses: (1) dual SEM-NIR fluorescence imaging and (2) single-particle tracking of EB-NS in a viscous glycerol solution. For the first approach, EB-NS were deposited on a glass cover slide with a grid. The labeled meshes enabled us to image the same nanostructures both under our NIR fluorescence imaging setup and under the SEM. As shown in Fig. 4a, it was thus possible to further confirm that diffraction-limited particles $<200 \mathrm{~nm}$ (long axis) can be imaged with our protocol. Smaller EB-NS could not be found in the SEM most likely because the contrast of a thin silicate nanosheet on glass $\left(\mathrm{SiO}_{2}\right)$ is too low and the material is non-conducting. However, scanning transmission electron microscopy (STEM) images showed EB-NS down to few tenths of nanometer in agreement with the AFM data (Supplementary Fig. 10).

In the second correlative approach, single EB-NS were imaged and their fluorescence intensity quantified in a viscous glycerol solution (chosen to slow down diffusion). This method allowed us to simultaneously quantify fluorescence intensity and Brownian motion as a measure of size of the same EB-NS. For this purpose, the particle trajectories were tracked and the mean square displacement (MSD, $\left\langle\mathbf{r}^{2}\right\rangle$ ) determined. We used the maximum value of the total integrated brightness of the tracked blob along each trajectory ("mass") as a measure of fluorescence intensity, to account for out-of-focus movement or rotations. A size equivalent can be derived from the trace of the random walk (Brownian motion) by employing the Stokes-Einstein equation. In Supplementary Figs. 11 and 12, trajectories and corresponding MSDs and NIR fluorescence images of exemplary EB-NS are shown. The trajectories (see Supplementary Fig. 11) show that the brighter particles move slower, indicating again that fluorescence intensity depends on size. To further investigate this finding, MSD curves were plotted and fitted: to estimate the EB-NS size, the fit was extended up to a certain lag time $\tau(25 \mathrm{~s})$ and the diffusion constant $(D)$

$$
D=\frac{\left\langle r^{2}\right\rangle}{4 \tau}
$$

was calculated. The Stokes radius $R$ is related to the diffusion constant via the Stokes-Einstein equation:

$$
R=\frac{k_{\mathrm{B}} T}{6 \pi \eta D}
$$

where $\eta$ is the dynamic viscosity of the solvent (Supplementary Fig. 13), $T$ the temperature (Supplementary Fig. 14), and $k_{\mathrm{B}}$ the Boltzmann constant. As EB-NS are rather anisotropic with a large aspect ratio (Figs. 1 and 3, and Supplementary Fig. 8), one could assume that diffusion is dominated by the diameter and not the height similar to a spherical particle. Approximations that correct the Stokes-Einstein equation for anisotropy have been used, e.g., to analyze carbon nanotube diffusion and length ${ }^{45}$. Similarly, we approximated the EB-NS by a nanometer-sized spheroid, inspired by the work of Happel et al. ${ }^{46}$, and confirmed the results by COMSOL simulations (see Supplementary Methods and Supplementary Fig. 15 for details). We calculated a correction factor of $A=1.49$ and corrected the results obtained from the Stokes-Einstein by this value leading to larger hydrodynamic radii. Using this method, both fluorescence intensity and hydrodynamic radii of single EB-NS are accessible (Fig. $4 \mathrm{~b}$ and Supplementary Figs. 16 and 17).

The sizes found in Fig. $4 \mathrm{~b}$ are in agreement with the AFMderived sizes in Fig. 1 but represent the (larger) hydrodynamic radii and not the actual physical particle radii (Supplementary Fig. 11). The results show again that larger EB-NS are brighter than smaller ones, indicating that fluorescence intensity correlates with the number of luminescent $\mathrm{Cu}^{2+}$ centers and thus the volume. The spread of data points along the fluorescence intensity axis at a given Stokes radius is most likely due to different layer numbers for sheets of similar diameter (and the already discussed out-of-focus issue as well). A power-law fit shows that fluorescence intensity scales with the square root of the diameter (Supplementary Fig. 16). However, future purification approaches that lead to samples of precise layer number and sheet diameter could increase the experimental accuracy and further enhance our understanding of how EB-NS fluorescence scales with dimension. Together with the photon counting experiments in Fig. 2, these results indicate that even the smallest EB-NS are fluorescent and fluorescence emission intensity correlates with the number of $\mathrm{Cu}^{2+}$ ions. Therefore, dimensionality does not appear to affect per se fluorescence properties beyond the change in the number of light-absorbing and -emitting $\mathrm{Cu}^{2+}$ centers.

Single-particle tracking of EB-NS in Drosophila melanogaster embryos. To demonstrate the potential of EB-NS for bioimaging applications, we performed single-particle tracking and microrheology measurements of EB-NS in embryos of the fruit fly D. melanogaster. This fly species is a widely employed model organism for studies ranging from fundamental genetics to developmental cell biology. During embryonal development the nuclei arrange in complex patterns mediated by microtubules and the actin cortex, but the underlying mechanisms and mechanics are poorly understood ${ }^{47}$. Following fertilization, the embryo develops as a syncytium ${ }^{47}$ in which the nuclei arrange in a regular 2D array linked to the actin cortex of the plasma membrane (Fig. 5a).

The dynamics of the nuclei and their associated centrosomes is determined by the cortical link and internuclear interactions 
a

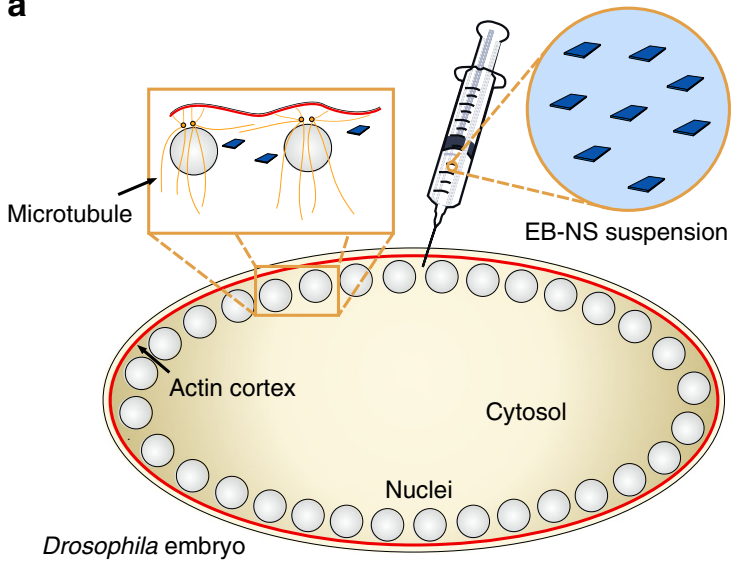

b

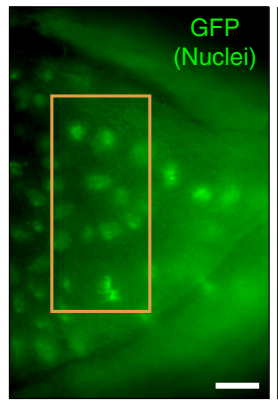

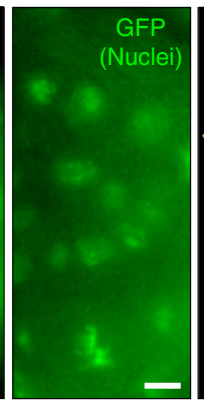
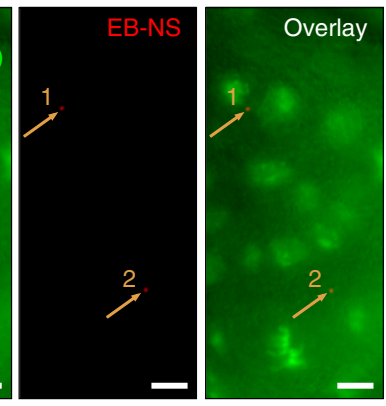
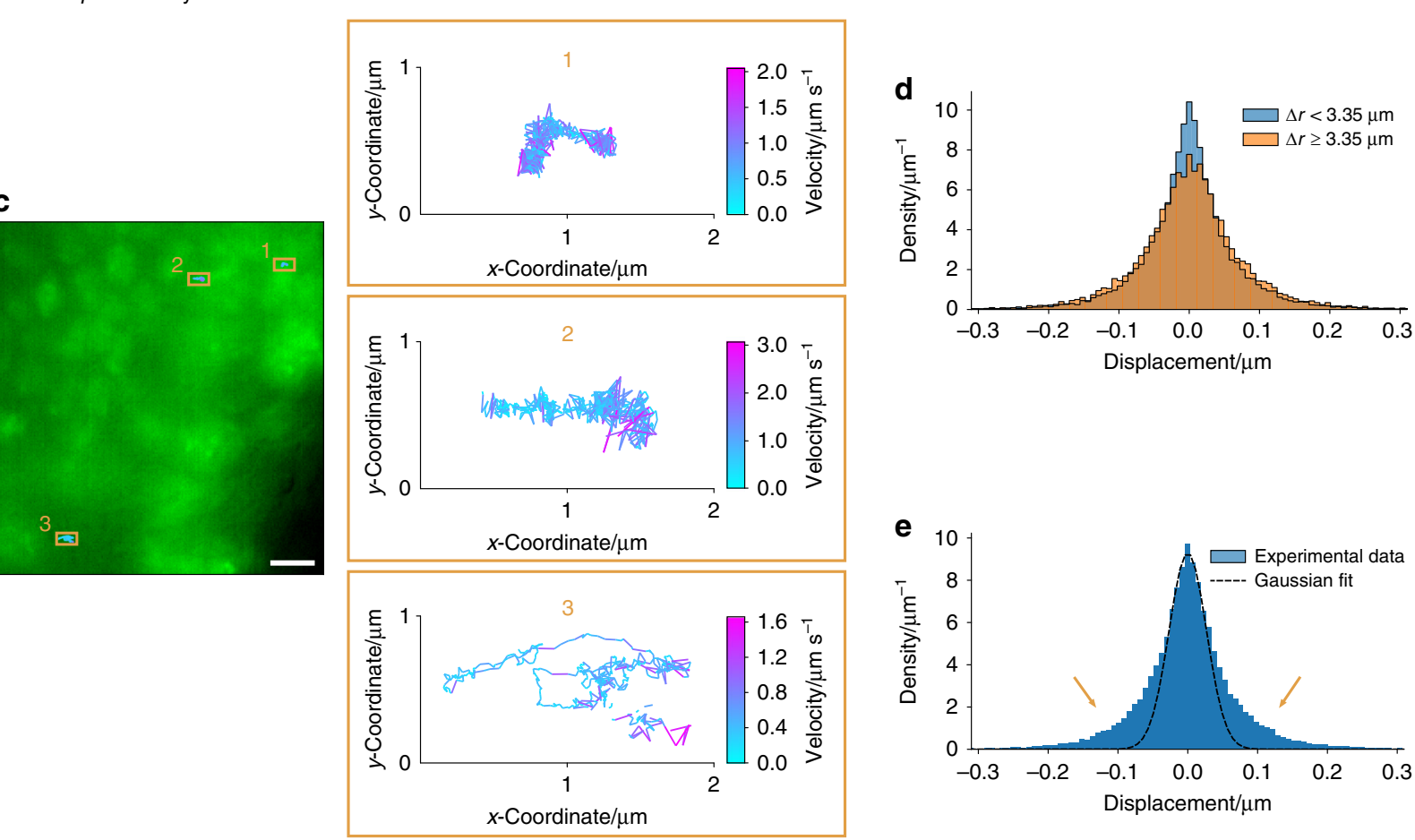

Fig. 5 In-vivo single-particle tracking and microrheology with EB-NS. a Experimental scheme for microinjection of EB-NS into syncytial Drosophila embryos. During the syncytial blastoderm stage, the peripheral nuclei are linked to the actin cortex of the plasma membrane (red). $\mathbf{b}$ EB-NS (red) inside a Drosophila embryo during gastrulation stage (stage 6) expressing Histone2Av-GFP (green) that labels the nuclei. Overview and magnified region (orange rectangle) with GFP (green), NIR EB-NS (red), and overlay channels are shown. Arrows pinpoint to the two EB-NS in this field of view. Scale bar $=10 \mu \mathrm{m}$ (overview) and $5 \mu \mathrm{m}$ (other channels). c Color-coded trajectories representing the instantaneous velocity of three tracked nanosheets moving in the space between the nuclei (lag time $=0.1 \mathrm{~s}$ ). Particles \#1, \#2, and \#3 have diameters (measured in the image) of $0.64 \mu \mathrm{m}, 0.68 \mu \mathrm{m}$, and $0.52 \mu \mathrm{m}$, and are most likely resolution-limited. Scale bar $=5 \mu \mathrm{m}$. $\mathbf{d}$ Van Hove histogram of displacements of two subpopulations of injected EB-NS: close to nuclei vs. distant from nuclei. The distance $(\Delta r)$ was computed for all points of all trajectories as the minimum distance to the border of any nucleus. e Van Hove plot of all tracked EB-NS and Gaussian fit for small displacements (range $[-0.03 \mu \mathrm{m},+0.03 \mu \mathrm{m}]$ ). The tails (see arrows) of the distribution exceed the Gaussian curve, which indicates the presence of active processes in Drosophila that lead to larger displacements than expected from thermal motion. $n=38$ tracked EB-NS (21 embryos).

mediated by microtubules ${ }^{48}$. It has been hypothesized that the viscoelastic properties and the molecular motors play a key role in this process ${ }^{47}$. To elucidate the micromechanical properties of the cellular matrix, we introduced EB-NS into Drosophila embryos and tracked single EB-NS via NIR fluorescence microscopy. Imaging in Drosophila is challenging due to autofluorescence in the visible region and its high sensitivity to phototoxicity; therefore, NIR imaging would be very beneficial.

For assessing the relative location of the EB-NS in Drosophila, mutants expressing green fluorescent protein (GFP)-labeled histones in their nuclei were used (Fig. 5b). Using this approach, we followed the traces of single EB-NS in vivo (Fig. 5b), allowing long-term imaging without bleaching or developmental abnormalities despite the high temporal resolution with frame rates of $10 \mathrm{~Hz}$. In addition, the low autofluorescence in this spectral range enabled a higher contrast of the NIR EB-NS image compared to the visible GFP image (Fig. 5b). These results highlight the great advantages of a non-bleaching NIR nanoscale fluorophore compared with typical organic dyes. To further prove the potential of EB-NS as NIR fluorophore for bioimaging, we were inspired by recent studies in which NIR fluorescent carbon nanotubes were used to explore the extracellular space between 
a

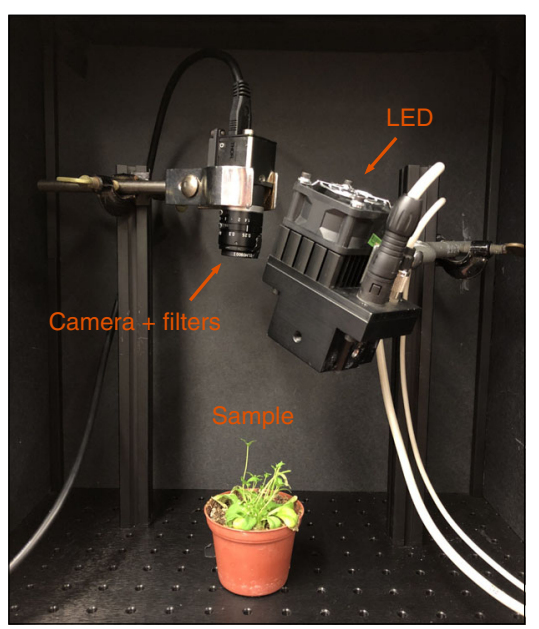

b
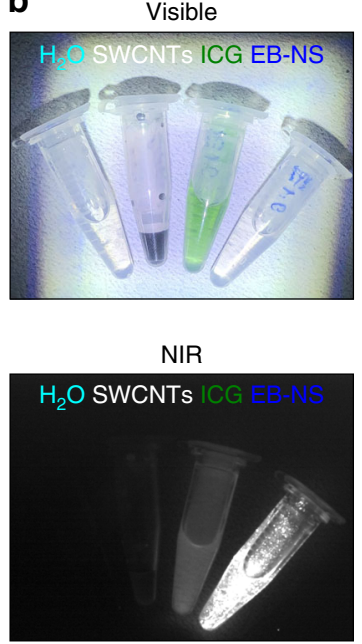

C

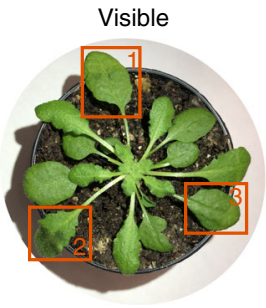

d

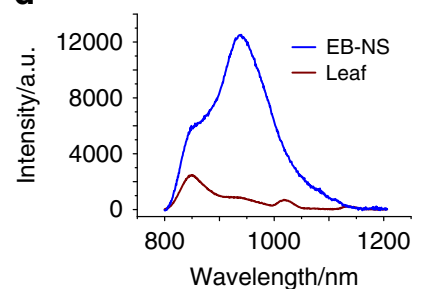

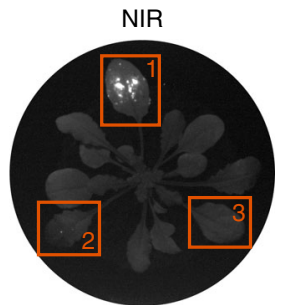

e NIR (room light)

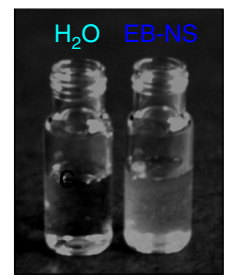

Fig. 6 Stand-off detection of EB-NS fluorescence in plants through low-cost and widely available imaging devices. a Photograph of a plant of Arabidopsis thaliana placed in a low-cost stand-off imaging system, which consisted of a LED, NIR filters and a Si-CMOS camera. b Visible and NIR fluorescence images of EB-NS $\left(\approx 0.1 \mathrm{mg} \mathrm{mL}^{-1}\right)$ compared with other NIR nanomaterials and fluorophores at similar concentration (single-walled carbon nanotubes (SWCNTs), indocyanine green (ICG)). Water is used as negative control. c Visible and NIR images of an Arabidopsis plant, which was infused with EB-NS (frame 1), SWCNTs (frame 2), and buffer only (frame 3). d The NIR fluorescence spectrum of the leaf confirms the presence of EB-NS and its strong emission compared to the leaf background. Both results demonstrate the high brightness of EB-NS compared to state-of-the-art NIR nanomaterials (SWCNTs) and that this platform can be applied for stand-off detection using a low-cost optical setup. e The EB-NS emission can even be detected without LED excitation under room light conditions.

neurons ${ }^{49}$. The instantaneous velocity of the injected EB-NS moving between nuclei was therefore analyzed (Fig. $5 \mathrm{c}$ and Supplementary Fig. 18). The goal was to use EB-NS as a probe for the extracellular space as a function of the distance to nuclei. To best display our results, established analysis tools such as the Van Hove histogram of displacements ${ }^{50}$ were chosen (Fig. $5 d$ ). The analysis revealed two subpopulations. EB-NS closer to nuclei showed smaller displacements, indicating a denser environment around the nuclei. EB-NS are likely to probe the mesh-size of the embryo's dense cytosol on the nanoscale and report mechanical properties on a different size scale than typical micrometer-sized beads used for microrheology ${ }^{47}$. Inside the embryo, one can expect active processes such as flow or motor-driven contractions ("active matter behavior"). Van Hove histograms provide access to test if there are such processes (Fig. 5e). The tails of the distribution go beyond the Gaussian function fit (thermal motion) and indicate the presence of active processes ${ }^{50}$. These could be, e.g., microtubules and kinesin motors (in the spindle close to the nuclei) that move the nuclei apart from each other and thereby lead to larger displacements of nearby EB-NS. Our results therefore show that EB-NS are powerful probes for studies of biological systems.

Biocompatibility and stand-off detection in living plants. In the context of biological applications, toxicity is a major concern and, e.g., a potential drawback of quantum dots that contain toxic elements and $2 \mathrm{D}$ materials in general ${ }^{51,52}$. To evaluate cytotoxicity, viability assays with EB-NS exposure to different cell lines (A549, 3T3, and MDCK-II) were performed. We observed no significant effects on the viability of these cell lines, highlighting the biocompatibility of EB-NS (Supplementary Fig. 19). In contrast, $\mathrm{Cu}^{2+}$ from soluble $\mathrm{CuSO}_{4}$ decreased cell viability, which shows that EB-NS does not release relevant $\mathrm{Cu}^{2+}$ ion concentrations as expected from a stable silicate. Overall, this study demonstrates that EB-NS are powerful and biocompatible nanoprobes in living organisms for single-particle tracking and microrheology measurements in the transparent NIR window.
Another potential application of NIR fluorescent EB-NS is non-contact stand-off detection of nanoprobes in living organisms with minimal perturbation. The low autofluorescence in the NIR region provides a high contrast, but stand-off detection requires especially bright fluorophores, because only a small portion of emitted light reaches the detector. Recently, NIR fluorescent SWCNT sensors enabled plants to report to electronic devices chemical signaling processes, pollutants in the environment ${ }^{53}$, and explosives ${ }^{54}$. Optical nanosensors are poised to allow the engineering of smart plants that communicate with and actuate agricultural and phenotyping devices for improving crop productivity and resource use $\mathrm{e}^{24}$.

Herein, we developed a low-cost optical setup to image the NIR emission of EB-NS from a distance $>10 \mathrm{~cm}$ and compared it with several other NIR fluorophores. The setup consisted of a white light LED and a camera $(1280 \times 1024$ pixels $)$ equipped with NIR (900-920 nm) long-pass filters (Fig. 6a) corresponding to $76 \mu \mathrm{m}$ pixel $^{-1}$. The high brightness of EB-NS allowed us to use a simple CMOS camera (Complementary Metal Oxide Semiconductor, Si-based) instead of expensive electrically or nitrogencooled InGaAs cameras ( $>40 \mathrm{k} €$ ), which would have a much higher quantum yield in this spectral range. Quantum yield of Sibased cameras decrease with increasing wavelength and therefore this low-cost setup favors fluorophores with lower emission wavelength. Therefore, ICG should appear slightly brighter and SWCNTs slightly dimmer than they really are and EB-NS would be in the middle. However, the SWCNT sample also contained $(6,4)$-SWCNTs that emit at $870 \mathrm{~nm}$. These different experimental conditions should be taken into account when assessing the performance of this low-cost setup. In the visible image (Fig. 6b), one can easily distinguish SWCNTs (black) from ICG (green), whereas the solution with EB-NS is transparent similar to the water control.

In contrast, in the corresponding NIR fluorescence image (Fig. 6b), EB-NS are significantly $(2 \times)$ brighter than ICG and $10 \times$ brighter than SWCNTs (Fig. 6b and Supplementary Fig. 20). Interestingly, stand-off detection of the NIR emission of EB-NS is also possible without LED excitation: room or sunlight alone is 
sufficient to generate detectable NIR fluorescence from EB-NS (Fig. 6e).

Buffered EB-NS were infused into the leaves of Arabidopsis thaliana, a well-established plant model system (Fig. 6c, d) ${ }^{53}$, using a standard method for nanomaterial delivery into plant leaves in-vivo ${ }^{53}$. The same approach was used for the infiltration of SWCNTs in separate leaves in the same plant, which served as a visual comparison with EB-NS. As it can be observed from the NIR image in Fig. 6c, plant leaves are characterized by a background autofluorescence in this wavelength region that hinders the visualization of SWCNTs fluorescence (frame 2) through a Si-based CMOS camera, whereas EB-NS can be detected with a strong fluorescence signal (frame 1). Similarly, fluorescence spectra of these leaves (Fig. 6d) show the strong EBNS emission compared to the (autofluorescence) background. These stand-off detection experiments highlight that EB-NS can be delivered into living organisms with high background fluorescence such as plants and can be detected using low-cost and widely available stand-off imaging devices.

\section{Discussion}

Our results show that EB-NS have useful properties for NIR bioimaging applications including ultra-high photostability, high brightness for NIR fluorophores, and biocompatibility. The remarkable properties of EB-NS are retained even when the dimensions of the nanosheets are reduced. Our data indicates that most if not all copper ions serve as luminescent centers that emit NIR light. It only affects the absorption cross-section by changing the number of light-absorbing $\mathrm{Cu}^{2+}$ ions. In contrast, the quantum yield of NIR fluorescent SWCNTs increases with length. As a consequence, shorter SWCNTs $(<100 \mathrm{~nm})$ are much less bright than longer ones ${ }^{40}$. Therefore, SWCNTs cannot be shortened without disadvantages. Although it has been reported that for bioimaging applications the NIR-II (1000-1700 nm) windows further improves tissue penetration ${ }^{5}$, a major advantage of the EB-NS fluorescence in the NIR-I region $(800-1000 \mathrm{~nm})$ is the wide availability of cameras ${ }^{55,56}$. Instead of expensive $(>40 \mathrm{k} €)$, liquid nitrogen-cooled, low-resolution InGaAs-based cameras, low-cost, $(1 \mathrm{k} €)$ high-resolution, Si-based cameras can be used for detection. In the past, $(6,4)$-SWCNTs with smaller diameter that emit in the NIR-I region at $870 \mathrm{~nm}$ have been isolated exactly for this purpose ${ }^{57}$. Even though the quantum yields of Si-based cameras rapidly decrease in the NIR (around $5 \%>900 \mathrm{~nm}$ ), they are still able to image EB-NS using a normal microscope or a lowcost stand-off detection device. Furthermore, there exist (bulk) pigment homologs Han Blue $\left(\mathrm{BaCuSi}_{4} \mathrm{O}_{10}\right)$ and Han Purple $\left(\mathrm{BaCuSi}_{2} \mathrm{O}_{6}\right)$, which have a red-shifted emission spectrum up to $1000 \mathrm{~nm}$, and they can be exfoliated into nanosheets using the same protocol as for EB (Supplementary Fig. 21). The capability of imaging EB-NS even with only room light excitation using Sibased cameras is particularly relevant for commercial applications beyond the well-equipped research laboratories or biomedical research, e.g., the engineering of smart plants for high-throughput phenotyping and precision agriculture. Another major advantage of EB-NS is their low toxicity, proven by the performed viability assays on cells (Supplementary Fig. 19). With our study, we add EB-NS to the library of 2D materials in general and especially exfoliated silicates and clays ${ }^{58,59}$. In the future, EB-NS and their physicochemical properties could be further investigated to find non-expected properties as in other $2 \mathrm{D}$ materials such as transition metal dichalcogenide nanosheets ${ }^{60}$. In addition, the surface chemistry could be tailored with biomolecules similar to silica nanoparticles and silicate-coated core-shell nanoparticles ${ }^{61,62}$.

In summary, we developed a method to exfoliate the calcium copper silicate EB into nanosheets (EB-NS) and demonstrated that their NIR optical properties are retained at the nanoscale with copper ions as luminescent centers. We add a promising 2D material to the class of NIR fluorophores with ultra-high photostability, high brightness and biocompatibility. EB-NS have a large potential for demanding biological applications such as invivo imaging in complex biological matrices, including animal and plant model systems.

\section{Methods}

Exfoliation of Egyptian Blue. EB powder was purchased from Kremer Pigmente $\mathrm{GmbH} \& \mathrm{Co}$. KG., and EB-NS were prepared as follows. If not stated otherwise, a first milling step was carried out. To produce sufficient amounts of nanoparticles, we used a PB mill (PB, Pulverisette 7 Premium Line, Fritsch, Germany) equipped with $20 \mathrm{~mL}$ agate beakers and $5 \mathrm{~mm}$ agate balls. The milling was performed in deionized water at 900 r.p.m. for up to $60 \mathrm{~min}$. The resulting grainsize distribution was analyzed by laser diffraction carried out with a LS13320, Beckman\&Coulter laser particle sizer (Germany). Three runs after a short tip sonication step were done. PIDS was used and an optical model R.I. 1.6/1 was applied. The so-generated and characterized EB slurry was afterwards allowed to settle down overnight and then decanted: only the supernatant portion of the slurry was dried and the soobtained powder (visibly brighter, a consequence of the dichroism of $\mathrm{EB}^{25}$ ) was finally processed with a long-tip sonication step. In more detail, $10 \mathrm{mg}$ of EB PBmilled powder was transferred to a glass vial together with $5 \mathrm{~mL}$ of isopropanol (Fischer Chemical, 99.98\%) or milli-Q water. Tip sonication was performed in a glass vial with a Fisherbrand ${ }^{m}$ Model 120 Sonic Dismembrator (Fischer Scientific) in an ice bath for 1-6h at 60-72 W (if not stated otherwise: typical tip sonication time and power percentage were $6 \mathrm{~h}$ and $60 \%$, respectively). EB dispersions were stored at room temperature and vortexed prior to use (Vortex Mixer VV3, VWR International) for $10 \mathrm{~s}$ at maximum power $\left(2500 \mathrm{~min}^{-1}, 10 \mathrm{~W}\right)$.

Atomic force microscopy. For this dataset, no PB milling step prior to tip sonication was performed. The pristine powder was ground with mortar and pestle till a visible color change could be observed, then it was tip sonicated (in isopropanol) as described in the previous section. One hundred microliters of EB-NS stock dispersion ( $6 \mathrm{~h}$ tip sonicated) were diluted $10 \times$ with isopropanol and vortexed for $10 \mathrm{~s}$. Ten microliters of EB-NS were spin-coated (G3 Spin Coater, Specialty Coating Systems, Inc.) on a mica surface. The substrate was kept spinning at 500 r.p.m. ( $7 \mathrm{RCF}$ ) for $2 \mathrm{~min}$ ( $5 \mathrm{~s}$ of ramp time, $30 \mathrm{~s}$ of dwell time). An Asylum Research MFP-3D Infinity AFM (Oxford Instruments) was employed in AC mode (software version 15.01.103). Rectangular cantilevers from Opus (160AC-NA, MikroMasch Europe) were used (aluminum coating, tetrahedral tip, $300 \mathrm{kHz}$ resonance frequency, force constant of $26 \mathrm{~N} \mathrm{~m}^{-1}$ ). Image analysis was performed with Gwyddion (version 2.51).

NIR fluorescence spectroscopy. The setup consists of a monochromator (MSH 150, LOT-Quantum Design $\mathrm{GmbH}$ ) equipped with a xenon arc lamp and a diffraction grating, an Olympus IX73 microscope with a 10× objective (UplanFLN $10 \times / 0.30$, Olympus), and a Shamrock 193i spectrograph (Andor Technology, Ltd) coupled to an array NIR detector (Andor iDUs InGaAs 491). Spectra were recorded from EB-NS dispersions at an excitation wavelength of $615 \mathrm{~nm}$ with an exposure time up to $5 \mathrm{~s}$ and a slit width up to $500 \mu \mathrm{m}$. The Andor SOLIS software (version 4.29.30012.0) was employed for the acquisition of spectra, which were and further analyzed with Origin Pro 8.1. For 2D spectra, EB-NS were placed on glass substrates. The excitation wavelength was scanned in $4 \mathrm{~nm}$ steps with the monochromator, and at each wavelength a spectrum was recorded with an integration time of $1 \mathrm{~s}$ at a slit width of $10 \mu \mathrm{m}$. The $2 \mathrm{D}$ spectra were corrected for the quantum efficiency of the detector and the spectral irradiance of the xenon lamp of the monochromator using a self-written Python script.

Fluorescence saturation measurements. The main components of the employed setup were a laser source (Supercontinuum laser SC400-4-20, Fianium), a photodetector (single photon avalanche diode PDM series, MPD), and a $60 \times$ objective lens (Apo N, 60×/1.49 numerical aperture (NA) oil immersion, Olympus). For this dataset, no PB milling step prior to tip sonication was performed. The pristine powder was ground with mortar and pestle till a visible color change could be observed, then it was tip sonicated (in isopropanol) as described above. Ten microliters of the supernatant of a $6 \mathrm{~h}$ tip-sonicated EB-NS sample (1:100 diluted in isopropanol) were spin-coated on a glass cover slide. Spin-coating parameters were the same ones chosen for AFM measurements. Despite the polydispersity of EBNS, scanning them using a confocal microscope through the diffraction-limited focal spot of 1.49 NA objective allowed us to select only the smallest particles with sizes estimated to be not exceeding the dimensions of the focal spot. The spot size of larger EB-NS exceeded the dimensions of a diffraction-limited focal spot, thus allowing us to distinguish them from smaller particles. After selecting a particle, we measured its fluorescence intensity at different excitation powers. Excitation of nanoparticles that have relatively long excited state lifetimes of the order of $100 \mu \mathrm{s}$ (Supplementary Fig. 7 ) with a laser that has high repetition rate $(20 \mathrm{MHz})$ allows 
one to extrapolate saturation of the nanoparticle's luminescence. The solid circles in Supplementary Fig. 6a show the dependence between the brightness of the particle and the excitation power that was measured directly before the objective lens. The excitation wavelength was $640 \mathrm{~nm}$. The fluorescence intensity values were normalized to the detection efficiency of the microscope, taking into account the average collection efficiency of light by the 1.49 NA objective lens ( $70 \%$, given the random orientation of the emission transition dipoles), transmissivity of all the optical elements involved ( 50\%) and detection efficiency of the single photon avalanche diode (PDM series, MPD) in the current spectral range ( 5\%). It is noteworthy that as a result of the above normalization, luminescence intensity in Fig. $2 \mathrm{~d}$ corresponds to the total number of photons emitted by the particle.

The experimental data were fitted with a typical fluorescence saturation function ${ }^{63}$ :

$$
I=I_{\text {sat }} \frac{P_{\text {exc }}}{P_{\text {exc }}+P_{\text {sat }}},
$$

where $P_{\text {exc }}$ is the excitation power, $P_{\text {sat }}$ is the saturation power, $I_{\text {sat }}$ is the nanoparticle luminescence intensity that can be detected at the saturation excitation power. This function yields the values of $P_{\text {sat }}$ and $I_{\text {sat }}$ for every saturation curve measured. Supplementary Table 1 shows the obtained values.

The obtained saturation luminescence intensity values allow one to estimate the number of luminescent centers $N$ per particle. Taking into account the average excited state lifetime $\tau(\sim 100 \mu \mathrm{s}$, Supplementary Fig. 7) and quantum yield $\Phi$ $\left(\sim 0.1^{28}\right)$, we recalculated the number of emitted photons $I_{\text {sat }}$ per second into the number of centers:

$$
N=\frac{I_{\text {sat }}}{\Phi \tau^{-1}}
$$

Fluorescence polarization measurements. The setup mainly consisted of a light source (LED, Lumencor), which emitted unpolarized excitation light, a photodetector (EMCCD camera, iXon Ultra DU-897U-CS0, Andor) and a $60 \times$ objective lens (Apo N, 60×/1.49 NA oil immersion, Olympus). Measurements were performed by recording fluorescence images of individual EB-NS at different positions on a linear polarizer, which was placed in front of the camera. The sample preparation steps coincided with the ones performed for fluorescence saturation measurements. Atto 488, purchased from Atto-Tec, was used as a reference.

NIR fluorescence imaging setup. An Olympus BX53 microscope equipped with

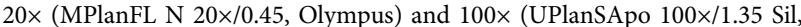
Olympus) objectives was used. A Xeva-1.7-320 NIR camera (Xenics ${ }^{\oplus}$ ) and a Zyla 5.5 sCMOS camera (Oxford Instruments) were used to observe the EB-NS fluorescence excited by a $561 \mathrm{~nm}$ laser (Cobolt Jive ${ }^{\mathrm{TM}} 561 \mathrm{~nm}$ ). Typically, a droplet of an EB-NS dispersion was put on glass slides, dried and imaged at $10-50 \mathrm{~mW}$ excitation power. To assess bleaching, a solution of Rhodamin B (Sigma-Aldrich) in isopropanol was prepared and a drop of this solution was put on a glass slide for imaging. Dried EB-NS were put on a separate glass slide. At a continuous excitation of $100 \mathrm{~mW}$ at $561 \mathrm{~nm}$, images with an integration time of $100 \mathrm{~ms}$ were recorded 120 times every $0.5 \mathrm{~min}$ in the case of Rhodamin B and every 1 min with an integration time of $1 \mathrm{~s}$ in the case of EB-NS. For Rhodamin B the Zyla camera was used and for EB the Xenics NIR camera was used. The specified fluorescence intensity corresponds to the mean gray value of the images.

For video-rate imaging and size-fluorescence correlation experiments, a modified setup equipped with a Cheetah 640TE3 camera (Xenics NV, Belgium) was used for NIR detection. Here, the light was passed through a dichroic mirror (HC BS R785, AHF, Germany) and a long-pass filter (FELH0900, Thorlabs, Inc., USA) before reaching the camera's sensor. Images were captured with the $100 \times$ objective.

Video-rate imaging of EB-NS with an InGaAs camera. EB-NS (previously milled and tip sonicated in isopropanol) underwent a single centrifugation step $(240 \times \mathrm{g}$ for $2 \mathrm{~h}$ ) to remove unexfoliated material. Ten microliters of EB-NS sample were spin-coated onto a \#1 glass coverslip with the following settings: 1000 r.p.m. (14 RCF) for $1 \mathrm{~min}$ ( $40 \mathrm{~s}$ of ramp time, $20 \mathrm{~s}$ of dwell time). The sample was then placed under the NIR imaging setup and pictures were taken with a $100 \times$ objective, laser power of $500 \mathrm{~mW}$ and exposure times in the range from $1 \mathrm{~s}$ to $5 \mathrm{~ms}$ (corresponding to frame rates ranging from 1 to $200 \mathrm{fps}$ ). Data analysis was performed with Image (v. 1.52a) and Origin Pro 8.1 software.

Scanning electron microscopy and SEM-NIR correlation. For a first qualitative observation of exfoliated EB-NS (Fig. 3a, b), no PB milling step prior to tip sonication was performed. The pristine powder was ground with mortar and pestle till a visible color change could be observed, then it was tip sonicated (in isopropanol) as described above. Ten microliters of EB-NS suspension were dropcasted on a Si wafer. Both gold sputtering and evaporation $(\approx 2 \mathrm{~nm}$ of gold layer $)$ were tested on different samples; nevertheless, the best imaging conditions were met when no gold deposition step was performed at all. Interestingly, EB was conductive enough to be seen at SEM without gold deposition. This sample was observed under a LEO SUPRA 35 microscope (Zeiss) with an Inlens detector at $20 \mathrm{kV}$ (secondary electrons).

For the SEM-NIR correlation, EB-NS (previously milled and tip sonicated in isopropanol) were centrifuged $(240 \times g$ for $2 \mathrm{~h})$. This way unexfoliated (bulk) material could be removed. Meanwhile, a \#1.5 gridded glass coverslip (Gridded Glass Coverslips Grid-50, Ibidi, Germany) was stirred for $20 \mathrm{~min}$ at $70^{\circ} \mathrm{C}$ in a 5 : $1: 1$ solution of milli-Q water : hydrogen peroxide : ammonia. Afterwards, the substrate was dried with a nitrogen gun and, to increase the adhesion of EB-NS on its surface, plasma treatment (Zepto, Diener Electronic GmbH +Co. KG, Germany) was performed: this step included $1 \mathrm{~min}$ of $\mathrm{O}_{2}$ supply and $1 \mathrm{~min}$ of plasma process. After cleaning the substrate as described, $10 \mu \mathrm{L}$ of EB-NS sample were spin-coated onto it with the following settings: 1000 r.p.m. (14 RCF) for 1 min ( $40 \mathrm{~s}$ of ramp time, $20 \mathrm{~s}$ of dwell time). The prepared sample was then observed at the NIR imaging setup (see corresponding section for more info) with a $100 \times$ objective, $1 \mathrm{~s}$ of exposure time, and $500 \mathrm{~mW}$ of laser power. Finally, the substrate was moved to the Quattro S SEM setup (Thermo Fisher Scientific, USA) employed for the size-fluorescence correlation analysis. Typical imaging settings here were: low vacuum mode, LVD detector (Thermo Fisher Scientific, USA), high voltage $=$ $15 \mathrm{kV}$ (secondary electrons), chamber pressure $=1.25 \mathrm{mbar}$ ( water), high voltage $=15 \mathrm{kV}$, and spot size $=3.5$. Data analysis was performed with ImageJ (v. 1.52a) and Origin Pro 8.1 software

Correlative size-intensity measurements in glycerol. EB-NS (previously milled and tip sonicated in water) were size-selected by means of two steps of liquid cascade centrifugation: ${ }^{33,64}$ in this way, we could get rid of unexfoliated EB and increase the monodispersity of the pellet obtained after the last step (first centrifugation $=240 \times g$ for $2 \mathrm{~h}$, second centrifugation $=2660 \times g$ for $2 \mathrm{~h}$ ). Glycerol $(0.5 \mathrm{~mL})$ (Alfa Aesar, $99+\%)$ was then added to the dried final pellet $(\approx 1 \mathrm{mg})$ and the sample was finally tip sonicated in an ice bath for $2 \mathrm{~min}$ at $60 \%$ to achieve a homogeneous redispersion. For imaging, $10-20 \mu \mathrm{L}$ of the glycerol sample were introduced into a flow chamber ( $\mu$-Slide VI 0.5 Glass Bottom, Ibidi, Germany) and placed under our NIR imaging setup. A 100× objective was employed to observe EB-NS at $8 \mathrm{fps}(\approx 50 \mathrm{~ms}$ exposure time, 1000 frames) with a laser power of $250 \mathrm{~mW}$, which, as shown in Supplementary Fig. 14, was not overheating the sample sig nificantly during acquisition. Particle tracking and MSD calculations were performed using a self-written Python script based on the Trackpy package ${ }^{65,66}$ (v0.4.2 on Python v3.7.367), whereas the final steps of data analysis were performed on Origin Pro 8.1 software. For identification and linking of particles into trajectories, the following parameters in Trackpy were implemented: memory $=300$, minimum number of points (i.e., trajectory length) $=300$, search_range (i.e., maximum displacement between consecutive frames) $=9$, diameter (of the tracked blob) $=11$. The tracking of the Brownian motion of an EB-NS yields the trajectory of the particle as a set of time-dependent $x$ and $y$ positions for $N$ time steps of length $\tau$. From the $x$ and $y$ positions, the square displacement for the $n$-th time step $\mathbf{r}_{n}^{2}$ is calculated as

$$
r_{n}^{2}=(x(n \cdot \tau)-x((n-1) \cdot \tau))^{2}+(y(n \cdot \tau)-y((n-1) \cdot \tau))^{2},
$$

where $n$ ranges from 1 to $N$. The MSD $\left\langle\mathbf{r}^{2}\right\rangle$ is simply the mean over all single time step values:

$$
\left\langle\mathbf{r}^{2}\right\rangle=\frac{1}{N} \sum_{n=1}^{N} \mathbf{r}_{n}^{2} .
$$

Assuming the diffusion is restricted to two dimensions, the diffusion coefficient $D$ and the MSD are linked via the relation

$$
D=\frac{r^{2}}{4 \tau} .
$$

MSD curves were fitted up to a maximum lag time of $25 \mathrm{~s}$, to measure the diffusion coefficient of each particle. Finally, from the Stokes-Einstein equation, the Stokes radius $R$ is calculated as

$$
R=\frac{k_{\mathrm{B}} T}{6 \pi \eta D},
$$

where $\eta$ is the dynamic viscosity of the solvent (Supplementary Fig. 13), $T$ the temperature (Supplementary Fig. 14), and $k_{\mathrm{B}}$ the Boltzmann constant. The Stokes-Einstein equation is only strictly valid for spherical particles. Due to the high anisotropy of EB-NS, we assumed that Brownian motion is dominated by the diameter of the nanosheets and not the much smaller height.

In-vivo microrheology of $\boldsymbol{D}$. melanogaster embryos. EB-NS (previously milled and tip sonicated in water) were filtered with a $0.20 \mu \mathrm{m}$ syringe filter. To further enhance the concentration of the smallest nanosheets in the sample, the filtered sample vial was placed in a concentrator (Eppendorf ${ }^{\circledR}$ centrifugal vacuum concentrator, Eppendorf, Germany) for $45 \mathrm{~min}$ at $45{ }^{\circ} \mathrm{C}$ and finally bath-sonicated for $10 \mathrm{~min}$ to reduce agglomeration. D. melanogaster embryos expressing Histone2Av$\mathrm{GFP}^{68}$ with an age of $0-1 \mathrm{~h}$ were collected and dechorionated with hypochlorite for $120 \mathrm{~s}$, washed with water thoroughly, aligned on a piece of agar, transferred to a coverslip coated with glue and covered with halocarbon oil (Voltalef $10 \mathrm{~S}$, Lehmann \& Voss) after a slight desiccation. An aliquot of suspension in water of the EB-NS 
sample was injected using Microinjector FemtoJet ${ }^{\circledR}$ (Eppendorf) on an inverted microscope: the injection volume is calculated according to literature ${ }^{69}$. After around $30 \mathrm{~min}$ of incubation, the sample could be moved to the NIR setup mentioned above for colocalization experiments. EB-NS were excited with the $561 \mathrm{~nm}$ laser (up to $500 \mathrm{~mW}$ ), whereas a fluorescence lamp (X-Cite ${ }^{\circledast}$ 120Q, Excelitas Technologies) was used for GFP excitation. Both channels were observed through a $100 \times$ objective and recorded with a Zyla 5.5 sCMOS camera. An exposure time of $0.1 \mathrm{~s}$ was chosen and images on both channels were taken for $60 \mathrm{~s}$ (10 fps). A $2 \times 2$ pixel binning was performed to lower the size of acquired data and thus facilitate the following particle tracking analysis. As for the EB-NS tracking in glycerol, custom-made scripts based on Trackpy were employed to analyze the acquired frames. Only the EB data were evaluated, since the nuclei did not display any significant motion during the acquisition. Typical parameters employed for particle tracking were: memory $=300$ frames (features vanishing for more than 300 frames were considered separate particles), minimum number of data points $=200$, maximum displacement between frames $=9$ pixels, and diameter (of the tracked blob) $=11$ pixels. The perimeter of the nuclei was manually measured on ImageJ and its coordinates implemented in a self-written Python code which could assess the minimum distance of each point along a trajectory to the closest nucleus perimeter. With a separate code, instantaneous velocity plots (lag time $=0.1 \mathrm{~s}$ ) could be evaluated and plotted. Van Hove histograms were plotted in Python, too, inspired by past works in literature ${ }^{50}$. The overlying Gaussian fit was performed for small displacements (range $[-0.03 \mu \mathrm{m},+0.03 \mu \mathrm{m}]$ ).

Stand-off detection. NIR images were acquired with a CMOS-based DCC $3240 \mathrm{M}$ camera (Thorlabs), equipped with a $900 \mathrm{~nm}$ (FEL0900, Thorlabs) and a $920 \mathrm{~nm}$ (LP920, Midwest Optical Systems) long-pass filter, mounted in series to exclude the visible background. A white light source (UHP, Prizmatix) connected to a $700 \mathrm{~nm}$ (FESH0700, Thorlabs) and a $750 \mathrm{~nm}$ (FESH0750, Thorlabs) short-pass filter was used for excitation. Exposure times between 50 and $100 \mathrm{~ms}$ were used for all acquisitions.

Single-stranded DNA (ssDNA)-modified SWCNTs were obtained by placing $125 \mu \mathrm{L}$ ( $2 \mathrm{mg} \mathrm{mL}^{-1}$ in PBS) (AT) $)_{15}$ ssDNA (Sigma-Aldrich) and $125 \mu \mathrm{L}(6,5)$ chirality enriched SWCNTs (Sigma-Aldrich, Product No. 773735) $\left(2 \mathrm{mg} \mathrm{mL}^{-1}\right.$ in PBS) for tip sonication (15 min, 30\% amplitude). The obtained suspension was centrifuged twice for $30 \mathrm{~min}$ at ambient temperature $(16100 \times g)$. The amounts of ICG $(\approx 90 \%$, MP Biomedicals $\mathrm{GmbH})$ and of a $6 \mathrm{~h}$ tip-sonicated sample of EB-NS in water were $\approx 0.1 \mathrm{mg} \mathrm{mL}^{-1}$.

Seeds of A. thaliana (Col.0 ecotype) were sown on sterilized soil $\left(8 \mathrm{~h}, 80^{\circ} \mathrm{C}\right)$ and stratified for 2 days in the dark at $4^{\circ} \mathrm{C}$. The plants were grown under long day length $\left(16 \mathrm{~h} \mathrm{light} / 8 \mathrm{~h}\right.$ dark) in climate-controlled growth chambers at $22^{\circ} \mathrm{C}$, $60 \%$ humidity, and light intensity of $120-150 \mu \mathrm{mol} \mathrm{m}^{-2} \mathrm{~s}^{-1}$. Delivery of nanomaterials and buffer into leaves of Arabidopsis was performed as described by Giraldo et al. ${ }^{53}$. In brief, $50 \mu \mathrm{l}$ of the desired NIR fluorophore suspension were infused through the lower (abaxial) side of the leaf lamina with a needleless syringe. Spectroscopic measurements for the presence of EB-NS in the leaf was performed using the NIR fluorescence spectroscopy setup $(5 \mathrm{~s}$ exposure time) as described above. Regarding the imaging of EB-NS under room light excitation, $1 \mathrm{~mL}$ of supernatant taken from a $6 \mathrm{~h}$ tip-sonicated sample in water was observed. A background picture was taken and used for background subtraction

Reporting summary. Further information on research design is available in the Nature Research Reporting Summary linked to this article.

\section{Data availability}

The data that support the findings of this study are available from the corresponding author upon reasonable request.

\section{Code availability}

The codes that support the findings of this study are available from the corresponding author upon reasonable request.

Received: 17 December 2019; Accepted: 28 February 2020;

Published online: 20 March 2020

\section{References}

1. Eggeling, C. et al. Direct observation of the nanoscale dynamics of membrane lipids in a living cell. Nature 457, 1159-1162 (2009).

2. Kruss, S. et al. High-resolution imaging of cellular dopamine efflux using a fluorescent nanosensor array. Proc. Natl Acad. Sci. USA 114, 1789-1794 (2017).

3. Smith, A. M., Mancini, M. C. \& Nie, S. Second window for in vivo imaging. Nat. Nanotechnol. 4, 710-711 (2009).
4. Owens, E. A., Henary, M., El Fakhri, G. \& Choi, H. S. Tissue-Specific NearInfrared Fluorescence Imaging. Acc. Chem. Res. 49, 1731-1740 (2016).

5. Hong, G., Antaris, A. L. \& Dai, H. Near-infrared fluorophores for biomedical imaging. Nat. Biomed. Eng. 1, 0010 (2017).

6. Carr, J. A. et al. Shortwave infrared fluorescence imaging with the clinically approved near-infrared dye indocyanine green. Proc. Natl Acad. Sci. USA 115 , $4465-4470$ (2018)

7. Zhu, S. et al. Molecular imaging of biological systems with a clickable dye in the broad 800- to 1,700-nm near-infrared window. Proc. Natl Acad. Sci. USA 114, 962-967 (2017).

8. Bruns, O. T. et al. Next-generation in vivo optical imaging with short-wave infrared quantum dots. Nat. Biomed. Eng. 1, 0056 (2017).

9. Fan, Y. et al. Lifetime-engineered NIR-II nanoparticles unlock multiplexed in vivo imaging. Nat. Nanotechnol. 13, 941-946 (2018).

10. He, S., Song, J., Qu, J. \& Cheng, Z. Crucial breakthrough of second nearinfrared biological window fluorophores: design and synthesis toward multimodal imaging and theranostics. Chem. Soc. Rev. 47, 4258-4278 (2018).

11. Hong, G., Diao, S., Antaris, A. L. \& Dai, H. Carbon nanomaterials for biological imaging and nanomedicinal therapy. Chem. Rev. 115, 10816-10906 (2015).

12. Kruss, S. et al. Carbon nanotubes as optical biomedical sensors. Adv. Drug Deliv. Rev. 65, 1933-1950 (2013).

13. Kruss, S. et al. Neurotransmitter detection using corona phase molecular recognition on fluorescent single-walled carbon nanotube sensors. J. Am. Chem. Soc. 136, 713-724 (2014).

14. Polo, E. \& Kruss, S. Impact of redox-active molecules on the fluorescence of polymer-wrapped carbon nanotubes. J. Phys. Chem. C. 120, 3061-3070 (2016).

15. Polo, E. et al. Control of integrin affinity by confining RGD peptides on fluorescent carbon nanotubes. ACS Appl. Mater. Interfaces 10, 17693-17703 (2018).

16. Bisker, G. et al. Protein-targeted corona phase molecular recognition. Nat. Commun. 7, 1-14 (2016)

17. Galassi, T. V. et al. An optical nanoreporter of endolysosomal lipid accumulation reveals enduring effects of diet on hepatic macrophages in vivo. Sci. Transl. Med. 10, 1-11 (2018).

18. Mann, F., Herrmann, N., Meyer, D. \& Kruss, S. Tuning selectivity of fluorescent carbon nanotube-based neurotransmitter. Sens. 17, 1521 (2017).

19. Zubkovs, V., Schuergers, N., Lambert, B., Ahunbay, E. \& Boghossian, A. A. Mediatorless, reversible optical nanosensor enabled through enzymatic pocket doping. Small 13, 1701654 (2017).

20. Nißler, R. et al. Quantification of the number of adsorbed DNA molecules on single-walled carbon nanotubes. J. Phys. Chem. C. 123, 4837-4847 (2019).

21. Mann, F. A. et al. Carbon nanotubes encapsulated in coiled-coil peptide barrels. Chem. A Eur. J. 24, 12241-12245 (2018).

22. Meyer, D., Hagemann, A. \& Kruss, S. Kinetic requirements for spatiotemporal chemical imaging with fluorescent nanosensors. ACS Nano 11, 4017-4027 (2017).

23. Fakhri, N. et al. High-resolution mapping of intracellular fluctuations using carbon nanotubes. Science 344, 1031-1035 (2014).

24. Giraldo, J. P., Wu, H., Newkirk, G. M. \& Kruss, S. Nanobiotechnology approaches for engineering smart plant sensors. Nat. Nanotechnol. 14, 541-553 (2019).

25. Warner, T. E. Synthesis, Properties and Mineralogy of Important Inorganic Materials (Wiley, 2011).

26. Ajò, D. et al. Photoluminescence of some blue natural pigments and related synthetic materials. In: 5th Int. Conf. on Non-Destructive Testing, Microanalytical Methods and Environmental Evaluation for Study and Conservation of Works of Art. 37-47 (1996).

27. Pozza, G., Ajò, D., Chiari, G., De Zuane, F. \& Favaro, M. Photoluminescence of the inorganic pigments Egyptian blue, Han blue and Han purple. J. Cult. Herit. 1, 393-398 (2000).

28. Accorsi, G. et al. The exceptional near-infrared luminescence properties of cuprorivaite (Egyptian blue). Chem. Commun. 3392-3394 https://doi.org/ 10.1039/b902563d (2009).

29. Johnson-McDaniel, D., Barrett, C. A., Sharafi, A. \& Salguero, T. T. Nanoscience of an ancient pigment. J. Am. Chem. Soc. 135, 1677-1679 (2013)

30. Johnson-McDaniel, D. \& Salguero, T. T. Exfoliation of Egyptian Blue and Han Blue, two alkali earth copper silicate-based pigments. J. Vis. Exp. 1-10 (2014).

31. Nicolosi, V., Chhowalla, M., Kanatzidis, M. G., Strano, M. S. \& Coleman, J. N. Liquid exfoliation of layered materials. Science 340, 1226419-1226419 (2013).

32. Cunningham, G. et al. Solvent exfoliation of transition metal dichalcogenides: dispersibility of exfoliated nanosheets varies only weakly between compounds ACS Nano 6, 3468-3480 (2012).

33. Backes, C. et al. Guidelines for exfoliation, characterization and processing of layered materials produced by liquid exfoliation. Chem. Mater. 29, 243-255 (2017). 
34. Coleman, J. N. et al. Two-dimensional nanosheets produced by liquid exfoliation of layered materials. Science 331, 568-571 (2011).

35. Wang, Q. H., Kalantar-Zadeh, K., Kis, A., Coleman, J. N. \& Strano, M. S. Electronics and optoelectronics of two-dimensional transition metal dichalcogenides. Nat. Nanotechnol. 7, 699-712 (2012).

36. Wu, S., Karius, V., Schmidt, B. C., Simon, K. \& Wörner, G. Comparison of ultrafine powder pellet and flux-free fusion glass for bulk analysis of granitoids by laser ablation-inductively coupled plasma-mass spectrometry. Geostand. Geoanal. Res. 42, 575-591 (2018).

37. Bachilo, S. M. et al. Structure-assigned optical spectra of single-walled carbon nanotubes. Science 298, 2361-2366 (2002).

38. Chen, Y., Kan, M., Sun, Q. \& Jena, P. Structure and properties of Egyptian Blue monolayer family: $\mathrm{XCuSi}_{4} \mathrm{O}_{10}(\mathrm{X}=\mathrm{Ca}, \mathrm{Sr}$, and $\mathrm{Ba})$. J. Phys. Chem. Lett. 7, 399-405 (2016).

39. Yang, S. S. et al. An exfoliated 2D Egyptian Blue nanosheet for highly selective enrichment of multi-phosphorylated peptides in mass spectrometric analysis. Chem. A Eur. J. 24, 2024 (2018).

40. Hertel, T., Himmelein, S., Ackermann, T., Stich, D. \& Crochet, J. Diffusion limited photoluminescence quantum yields in 1-D semiconductors: singlewall carbon nanotubes. ACS Nano 4, 7161-7168 (2010).

41. Sikora, A. et al. A systematic comparison of different techniques to determine the zeta potential of silica nanoparticles in biological medium. Anal. Methods 7, 9835-9843 (2015).

42. Chaze, W., Caballina, O., Castanet, G. \& Lemoine, F. The saturation of the fluorescence and its consequences for laser-induced fluorescence thermometry in liquid flows. Exp. Fluids 57, 58 (2016).

43. Pabst, A. Structures of some tetragonal sheet silicates. Acta Crystallogr. 12, 733-739 (1959).

44. Li, Y. J., Ye, S., Wang, C. H., Wang, X. M. \& Zhang, Q. Y. Temperaturedependent near-infrared emission of highly concentrated $\mathrm{Cu} 2+$ in CaCuSi4O10 phosphor. J. Mater. Chem. C. 2, 10395-10402 (2014).

45. Streit, J. K. et al. Measuring single-walled carbon nanotube length distributions from diffusional trajectories. ACS Nano 6, 8424-8431 (2012).

46. Happel, J. \& Brenner, H. The motion of a rigid particle of arbitrary shape in an unbounded fluid. Low Reynolds Number Hydrodyn. 159-234 (1983).

47. Wessel, A. D., Gumalla, M., Grosshans, J. \& Schmidt, C. F. The mechanical properties of early Drosophila embryos measured by high-speed video microrheology. Biophys. J. 108, 1899-1907 (2015).

48. Winkler, F. et al. Fluctuation analysis of centrosomes reveals a cortical function of Kinesin-1. Biophys. J. 109, 856-868 (2015).

49. Godin, A. G. et al. Single-nanotube tracking reveals the nanoscale organization of the extracellular space in the live brain. Nat. Nanotechnol. 12, 238-243 (2017).

50. Toyota, T., Head, D. A., Schmidt, C. F. \& Mizuno, D. Non-Gaussian athermal fluctuations in active gels. Soft Matter 7, 3234-3239 (2011).

51. Zhao, J., Zhong, D. \& Zhou, S. NIR-I-to-NIR-II fluorescent nanomaterials for biomedical imaging and cancer therapy. J. Mater. Chem. B 6, 349-365 (2018).

52. Martín, C., Kostarelos, K., Prato, M. \& Bianco, A. Biocompatibility and biodegradability of 2D materials: graphene and beyond. Chem. Commun. 55, 5540-5546 (2019).

53. Giraldo, J. P. et al. Plant nanobionics approach to augment photosynthesis and biochemical sensing. Nat. Mater. 13, 400-408 (2014).

54. Wong, M. H. et al. Nitroaromatic detection and infrared communication from wild-type plants using plant nanobionics. Nat. Mater. 16, 264-272 (2017).

55. Hong, G. et al. Multifunctional in vivo vascular imaging using near-infrared II fluorescence. Nat. Med. 18, 1841-1846 (2012).

56. Hong, G. et al. Through-skull fluorescence imaging of the brain in a new nearinfrared window. Nat. Photonics 8, 723-730 (2014).

57. Antaris, A. L. et al. Single chirality $(6,4)$ single-walled carbon nanotubes for fluorescence imaging with silicon detectors. Small 11, 6325-6330 (2015).

58. Stöter, M., Rosenfeldt, S. \& Breu, J. Tunable exfoliation of synthetic clays. Annu. Rev. Mater. Res. 45, 129-151 (2015).

59. Tao, H. et al. Scalable exfoliation and dispersion of two-dimensional materials - an update. Phys. Chem. Chem. Phys. 19, 921-960 (2017).

60. Chhowalla, M. et al. The chemistry of two-dimensional layered transition metal dichalcogenide nanosheets. Nat. Chem. 5, 263-275 (2013).

61. Parak, W. J. et al. Conjugation of DNA to silanized colloidal semiconductor nanocrystalline quantum dots. Chem. Mater. 14, 2113-2119 (2002)

62. Ow, H. et al. Bright and stable core-shell fluorescent silica nanoparticles. Nano Lett. 5, 113-117 (2005).

63. Plakhotnik, T. \& Gruber, D. Luminescence of nitrogen-vacancy centers in nanodiamonds at temperatures between 300 and $700 \mathrm{~K}$ : Perspectives on nanothermometry. Phys. Chem. Chem. Phys. 12, 9751-9756 (2010).
64. Backes, C. et al. Production of highly monolayer enriched dispersions of liquid-exfoliated nanosheets by liquid cascade centrifugation. ACS Nano 10 1589-1601 (2016)

65. Crocker, J. C. \& Grier, D. G. Methods of digital video microscopy for colloidal studies. J. Colloid Interface Sci. 179, 298-310 (1996).

66. Allan, D. et al. soft-matter/trackpy: Trackpy v0.4.2. https://doi.org/10.5281/ ZENODO.3492186 (2019).

67. https://www.python.org/.

68. Gramates, L. S. et al. FlyBase at 25: looking to the future. Nucleic Acids Res. 45, D663-D671 (2017).

69. Markow, T. A., Beall, S. \& Matzkin, L. M. Egg size, embryonic development time and ovoviviparity in Drosophila species. J. Evol. Biol. 22, 430-434 (2009).

\section{Acknowledgements}

We thank the Volkswagen foundation and the life@nano cluster for funding (S.K.). We thank Dr. Burkhard Schmidt for recording reflectance spectra; Dr. Elena Polo for initial exfoliation experiments; Dr. Nelli Teske, Dr. Jeremias Sibold, and Dr. Ingo Mey for help in the acquisition of SEM images; Angela Rübeling for expert technical assistance; Wentao Peng for assistance with zeta potential measurements; and Dr. Ellen Hornung for providing us with Arabidopsis plants. Furthermore, we thank Dr. Florian Rehfeldt for viscosity measurements, Dr. Heidrun Sowa for input on diffractometry, and Jonathan Bodenschatz and Nils Liebe for support in IR monitoring measurements and SEM sample preparation. Some experimental work was done in collaboration with the department of cell culture, Institute for Organic and Biomolecular Chemistry, GeorgAugust-University Göttingen. Parts of this work was funded by the National Science Foundation under Grant Number 1817363 to J.P.G.

\section{Author contributions}

S.K. conceived and designed the study. G.S. and V.K. performed and optimized the milling procedure. G.S. performed AFM experiments. G.S., H.P., and A.S. exfoliated materials and collected 1D spectra. H.P. and R.N. collected 2D spectra. A.C. performed single photon counting and anisotropy experiments. G.S. and L.V. performed and analyzed correlative SEM-NIR experiments. G.S. imaged single nanosheets and analyzed particle trajectories together with N.H., I.K., and A.J. G.S. together with R.N., F.A.M., and Z.L. performed biological imaging experiments with input from L.E., J.P.G., and J.G.. T.A.O. performed cell viability experiments. D.M. performed calculations and simulations concerning the correction factor for diffusion of (anisotropic) EB-NS in a solvent. G.S. and S.K. wrote the manuscript with contributions from all authors.

\section{Competing interests}

The authors declare no competing interests.

\section{Additional information}

Supplementary information is available for this paper at https://doi.org/10.1038/s41467020-15299-5.

Correspondence and requests for materials should be addressed to S.K.

Peer review information Nature Communications thanks Eleni Stavrinidou and the other anonymous reviewer(s) for their contribution to the peer review of this work. Peer reviewer reports are available.

Reprints and permission information is available at http://www.nature.com/reprints

Publisher's note Springer Nature remains neutral with regard to jurisdictional claims in published maps and institutional affiliations.

\footnotetext{
Open Access This article is licensed under a Creative Commons Attribution 4.0 International License, which permits use, sharing, adaptation, distribution and reproduction in any medium or format, as long as you give appropriate credit to the original author(s) and the source, provide a link to the Creative Commons license, and indicate if changes were made. The images or other third party material in this article are included in the article's Creative Commons license, unless indicated otherwise in a credit line to the material. If material is not included in the article's Creative Commons license and your intended use is not permitted by statutory regulation or exceeds the permitted use, you will need to obtain permission directly from the copyright holder. To view a copy of this license, visit http://creativecommons.org/ licenses/by/4.0/.
}

(c) The Author(s) 2020 